\title{
FOCUS OF ATTENTION AND REGION SEGREGATION BY LOW-LEVEL GEOMETRY
}

\author{
J.A. Martins, J. Rodrigues and J.M.H. du Buf \\ Vision Laboratory, Institute for Systems and Robotics (ISR), University of the Algarve, Campus de Gambelas, 8005-139 Faro, Portugal \\ \{jamartins, jrodrig, dubuf\}@ualg.pt
}

Keywords: $\quad$ Saliency, Focus-of-Attention, Region Segregation, Colour, Texture.

\begin{abstract}
Research has shown that regions with conspicuous colours are very effective in attracting attention, and that regions with different textures also play an important role. We present a biologically plausible model to obtain a saliency map for Focus-of-Attention (FoA), based on colour and texture boundaries. By applying grouping cells which are devoted to low-level geometry, boundary information can be completed such that segregated regions are obtained. Furthermore, we show that low-level geometry, in addition to rendering filled regions, provides important local cues like corners, bars and blobs for region categorisation. The integration of FoA, region segregation and categorisation is important for developing fast gist vision, i.e., which types of objects are about where in a scene.
\end{abstract}

\section{INTRODUCTION}

Attention of animals, also primates and humans, is rapidly drawn towards conspicuous objects and regions in the visual environment. The ability to identify such objects and regions in complex and cluttered environments is key to survival, for locating possible prey, predators, mates or landmarks for navigation (Elazary and Itti, 2008). But attention is only one aspect. We start to understand how our visual system works: (1) very fast extraction of global scene gist, (2) also fast local gist for important objects and a rough spatial layout map, (3) in parallel with (2) the construction of a saliency map for Focus-of-Attention (FoA), and only then (4) sequential screening of conspicuous regions for precise object recognition, using peaks and regions in the saliency map with inhibitionof-return in order not to fixate the same region twice, but with two strategies for FoA: first covert attention (automatic, data-driven) possibly followed by overt attention (consciously directed). In addition, our visual system is not analysing all information for constructing a complete and detailed map of our environment; it concentrates on essential information for the task at hand and it relies on the physical environment as external memory (Rensink, 2000).
In this paper we concentrate on three aspects: (1) the construction of a saliency map for FoA on the basis of colour, which was shown to be very effective in attracting attention (van de Weijer et al., 2006), also texture (du Buf, 2007), (2) a first region segregation by employing low-level geometry in terms of blobs, bars and corners, and (3) using low-level geometry allows us to reduce significantly the dimensionality of texture features. We note that our approach is not based on the cortical multi-scale keypoint representation as recently proposed by Rodrigues and du Buf (2006), who built saliency maps which work very well for the detection of facial landmarks and for invariant object recognition on homogeneous backgrounds (Rodrigues and du Buf, 2007), but may lead to enormous amounts of local peaks in natural scenes.

\section{COLOUR CONSPICUITY}

Colour information in a saliency map was first used by Niebur and Koch (1996). Their model was later extended by Itti and Koch (2001), who integrated more features, for instance intensity, edge orientation and motion. In our approach to create a saliency model which also contains cues for region and object segregation, we therefore start by using colour information, 
as this provides the most important input for attention (van de Weijer et al., 2006), in order to build a colour conspicuity map which will later be combined with a texture map. But before using colour features the input images must be corrected because a same object will look different when illuminated by different light sources, i.e., the number, power and spectra of these.

The processing consists of the following four steps: (a) colour illuminant and geometry normalisation deals with correcting the image's colours. Let each pixel $P_{i}$ of image $I(x, y)$ be defined as $\left(R_{i}, G_{i}, B_{i}\right)$ and $\left(L_{i}, a_{i}, b_{i}\right)$ in both RGB and Lab colour spaces, with $i=\{1 \ldots N\}, N$ being the total number of pixels in the image. We first process the input image $I_{\text {in }}$ using the two transformations described by Finlayson et al. (1998), as shown below, both in RGB colour space. Their method applies iteratively steps A and B, until colour convergence is achieved (4-5 iterations). Each individual pixel is first corrected in step A for illuminant geometry independency (i.e., chromaticity), by

$$
P_{i}^{A}=\left(\frac{R_{i}}{R_{i}+G_{i}+B_{i}}, \frac{G_{i}}{R_{i}+G_{i}+B_{i}}, \frac{B_{i}}{R_{i}+G_{i}+B_{i}}\right),
$$

followed in step B by global illuminant colour independency (i.e., grey-world normalisation),

$$
P_{i}^{B}=\left(\frac{N \cdot R_{i}}{\sum_{j=1}^{N} R_{j}}, \frac{N \cdot G_{i}}{\sum_{j=1}^{N} G_{j}}, \frac{N \cdot B_{i}}{\sum_{j=1}^{N} B_{j}}\right) .
$$

After the process is completed, the resulting RGB image is converted to Lab colour space and the $a_{c c}$ and $b_{c c}$ components, where subscript $c c$ stands for colourcorrected, are combined in $I_{c c}$ together with the unmodified $L_{\text {in }}$ channel from the input image $I_{i n}$. The main idea for using the Lab space is that it is an almost linear colour space, i.e., it is more useful for determining the conspicuity of borders between regions. The reason for using the $L_{i n}$ component instead of the $L_{c c}$ one is that, as observed by Finlayson et al. (1998), the simple and fast repetition of steps A and $\mathrm{B}$ does a remarkably good job. In fact, it does the job too well because all gray pixels (with values $R=G=B$ from 0 to 255) end up having $R=G=B=127$. In other words, all information in gray image regions would be lost. Summarising, the initial $I_{\text {in }}$ image in RGB is normalised to $I_{c c}$ and then converted to the colour space $L_{i n} a_{c c} b_{c c}$.

Figure 1 shows four input images which will be used below, called extinguisher, park, fish and mountain, all of size $256 \times 256$ pixels with 8 bits for each colour component R, G and B. Figure 2 shows three results of colour correction applied to the extinguisher image, from top-left to top-right: original image, modified image with a blue tint $(\mathrm{R}-12 \%, \mathrm{G}$ $+4 \%$ and $\mathrm{B}+50 \%$ ), and modified image with a warm

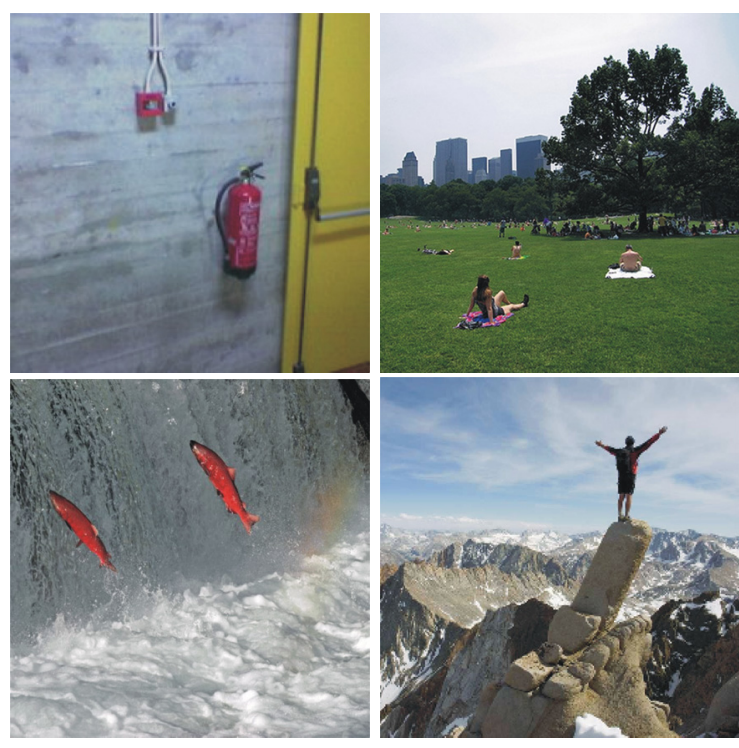

Figure 1: Input images, left to right and top to bottom: $e x$ tinguisher, park, fish and mountain.

white balance. The three results are shown below the input images. As can be seen, colour correction yields very similar images despite the rather large differences in the input images. Colour correction as explained above simulates colour constancy as employed in our visual system (Hubel, 1995).

The second step (b) is to reduce colour inhomogeneities in the images by adaptive smoothing of the colour regions, while maintaining or even improving the boundaries between different regions. We propose a new, nonlinear, adaptive 1D filter, here explained in the horizontal direction but it can be rotated, which consists of a centred DOG

$$
F_{1,2}(x)=N_{1}\left\{\exp \left(\frac{-x^{2}}{2 \sigma_{1}^{2}}\right)-\exp \left(\frac{-x^{2}}{2 \sigma_{2}^{2}}\right)\right\},
$$

which is split into $F_{1}(x<0)$ and $F_{2}(x>0)$, and another centred Gaussian, which is not split,

$$
F_{3}(x)=N_{2} \cdot \exp \left(\frac{-x^{2}}{2 \sigma_{2}^{2}}\right),
$$

taking $\sigma_{1} \gg \sigma_{2} . N_{1}$ and $N_{2}$ are normalisation constants which make the integrals of all three functions equal to one. The three functions can be seen as a simulation of a group of three cells at the same position, but with different dendritic fields which are indirectly connected to cone receptors in the colouropponent channels a and $\mathrm{b}$ of Lab, $F_{3}$ yielding the excitatory response of a receptive field of an oncentre cell, and $F_{1,2}$ yielding excitatory responses of two off-centre cells. From the three cell responses $R_{1,2,3}$ we first compute the contrast between the left $\left(R_{1}\right)$ and right $\left(R_{2}\right)$ responses; mathematically $C=$ 

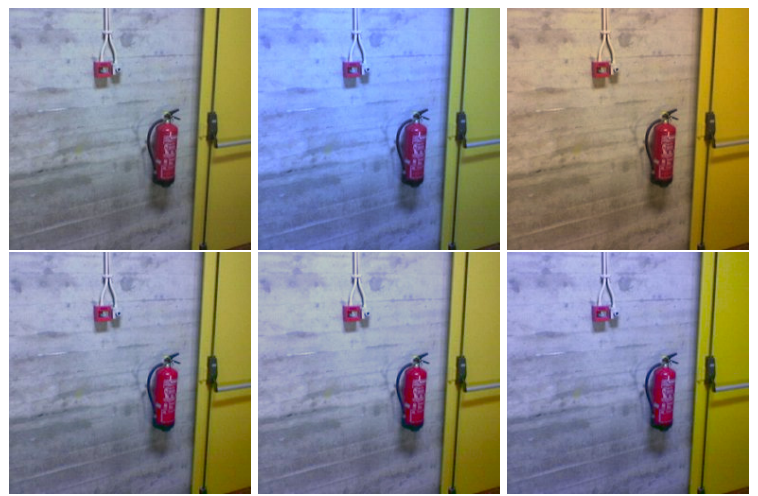

Figure 2: Colour illuminant and geometry normalisation. Top: input images; bottom: respective results; see text.

$\left|\left(R_{1}-R_{2}\right) /\left(R_{1}+R_{2}\right)\right|$. Then, based on the contrast $C$ and the minimum difference between the centre response $\left(R_{3}\right)$ and the left and right responses, the output $R$ is determined by

$R= \begin{cases}C R_{1}+(1-C) R_{3} & \text { if }\left|R_{1}-R_{3}\right|<\left|R_{2}-R_{3}\right| \\ C R_{2}+(1-C) R_{3} & \text { otherwise. }\end{cases}$

In words, if the contrast is low, as in an almost homogeneous region, the filter support is big, but if the contrast is high, at the boundary between two regions, the filter support is small. This adaptive filtering is applied to $I_{c c}$ at each pixel position $(x, y)$, first horizontally $\left(R^{H}\right)$ and then vertically $\left(R^{V}\right)$ :

$$
I_{c i}(x, y)=R^{V}\left[R^{H}\left[I_{c c}(x, y)\right]\right],
$$

where subscript $c i$ stands for colour-improved. In our experiments we obtained good results with $\sigma_{1}=7$ and $\sigma_{2}=3$ ), and adaptive filtering in horizontal and vertical directions was sufficient to sharpen blurred boundaries even with oblique orientations. Furthermore, the processing is very fast because the three filter functions need only be computed once.

After colour correction and adaptive filtering, the third step (c) serves to detect boundaries. In fact, for this purpose we could use the contrast function $C$ described above, but in order to accelerate processing we apply a simple gradient operator, as shown in Fig. $3 \mathrm{a}$ and $\mathrm{b}$, which requires only two convolutions, with mask sizes of $5 \times 2$ and $2 \times 5$, of the components of $I_{c i}$. These masks can be seen as dendritic fields of two cells, the two results being subtracted by a third cell which combines horizontal and vertical gradients:

$$
\begin{aligned}
\widehat{I}_{e d}(x, y) & =\sum_{\text {left }} I_{c i}(x, y)-\sum_{\text {right }} I_{c i}(x, y) \\
& +\sum_{\text {top }} I_{c i}(x, y)-\sum_{\text {bottom }} I_{c i}(x, y) .
\end{aligned}
$$

$\widehat{I}_{e d}(x, y)$ is then thresholded using

$$
I_{e d}(x, y)= \begin{cases}1 & \text { if } \widehat{I}_{e d}(x, y)>k \\ 0 & \text { otherwise, }\end{cases}
$$

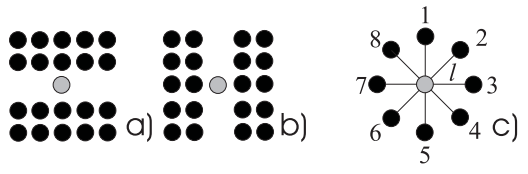

Figure 3: Gradient operators in vertical (a) and horizontal (b) orientations with summation areas of $2 \times 5$ pixels, and (c) the cluster of gating cells used for colour conspicuity.

where subscript ed stands for edge-detected. This yields a binary edge map by means of a cell layer in which cells are either active (response 1) or inactive (response 0). We apply a global threshold $k=\max \left(I_{c i}(x, y)\right)$. Edge detection yields three distinct maps, one for each component of Lab colour space, $I_{e d}^{L}, I_{e d}^{a}$ and $I_{e d}^{b}$, which can be combined.

In the last step (d), colour conspicuity at colour edges is calculated at each position in the edge map where there is an active cell $\left(I_{e d}^{L, a, b}(x, y)=1\right)$. We define conspicuity $\Psi$ at position $(x, y)$ as the maximum difference between the colours in $I_{c i}$ at four pairs of symmetric points at distance $l$ from $(x, y)$, i.e., on horizontal, vertical and two diagonal lines. Figure $3 \mathrm{c}$ shows a cluster of gating cells. If the gating cells are called $G_{i}$, opposing pairs are $\left(G_{i}, G_{i+4}\right)$, with $i=\{1, \ldots, 4\}$, for example $\left(G_{1}, G_{5}\right)$. Partial conspicuity is then calculated independently for each of the colour components in Lab space, as defined by (9a), where $\vec{x}_{i}$ denotes the position of $G_{i}$ relative to position $(x, y)$. The final value is then calculated using the sum of all three colour components (9b).

$$
\begin{aligned}
\Psi_{L, a, b}(x, y) & =\max _{i}\left(\left|I_{c i}^{L, a, b}\left(\vec{x}_{i}\right)-I_{c i}^{L, a, b}\left(\vec{x}_{i+4}\right)\right|\right), \\
\Psi_{L a b}(x, y) & =\Psi_{L}(x, y)+\Psi_{a}(x, y)+\Psi_{b}(x, y) .
\end{aligned}
$$

Results of colour conspicuity are shown in Fig. 4 top, for the park and mountain images, using $l=4$.

\section{TEXTURE BOUNDARIES}

Colour conspicuity $\Psi_{L a b}$ includes the luminance component $\mathrm{L}$ and therefore luminance gradients, both in coloured image regions and in non-coloured or gray ones, but the processing as applied up to here is too local to capture texture as a region property. As different colours in surrounding or neighbouring regions attract attention, so do different textures because texture conveys complexity and therefore importance of regions to attend for screening.

Texture processing is in principle completely equal to colour processing, with adaptive filtering, gradient detection and the attribution of conspicuity to texture boundaries, but instead of using the three Lab components only the $\mathrm{L}$ one is used and texture features must be extracted from $L(x, y)$. Since we are developing biologically plausible methods, it makes 
sense to apply Gabor wavelets as a model of cortical simple cells. Although very sophisticated texture models have been proposed on the basis of the Gabor model (du Buf, 2007), we will only use the spectral decomposition here because of speed. This frees CPU time for applying a reasonable number of frequency (scale) and orientation channels, which will be $8 \times 8=64$ in this paper. Since Gabor filtering involves filter kernels which are relatively small (highfrequency textures because of viewing distance), all filtering can be done in the frequency domain (see e.g. Rodrigues and du Buf (2004)) and requires one forward FFT and 64 inverse FFTs, the latter parallelised on multi-core CPUs or even graphics boards (GPUs).

In the spatial domain, Gabor filters consist of a real cosine and an imaginary sine component, both with a Gaussian envelope, which resemble receptive fields of simple cells with even $R_{s, i}^{E}$ and odd symmetry $R_{s, i}^{O}$, with $i$ the orientation and $s$ the scale. Responses of complex cells are modelled by taking the modulus $C_{s, i}(x, y)=\left[\left\{R_{s, i}^{E}(x, y)\right\}^{2}+\left\{R_{s, i}^{O}(x, y)\right\}^{2}\right]^{\frac{1}{2}}$.

Texture boundaries are obtained by applying three processing steps to the responses of complex cells, at each individual scale and orientation, after which results are combined: (a) the responses $C_{s, i}(x, y)$ are smoothed using the adaptive filter defined by eqns (3) to (5), obtaining $\widehat{C}_{s, i}$. The next step (b) consists of horizontal and vertical gradient detection $\bar{C}_{s, i}$, applying cells with dendritic fields of size $2 \times 5$ as shown in Fig. 3a, b to $C_{s, i}(x, y)$. The final step (c) consists of summing the results at all scales and orientations

$$
R(x, y)=\sum_{s, i} \bar{C}_{s, i}(x, y),
$$

together with an inhibition of all responses below a threshold (we apply $0.1 \max \{R(x, y)\}$ ). Figure 4 (bottom) shows the results in the case of park and mountain. As can be seen, the information is more diffuse and complements that of colour processing (top).

\section{SALIENCY MAP}

A saliency map is built on top of colour conspicuity and texture boundary maps by using grouping cells which code local geometry. There are two levels of grouping cells. At the first, lower level, there are summation cells with a dendritic field size of $n \times m$, with the centres at a distance $d$; see Fig. 5a. In this paper we use $m=n=5$ and $d=5$, such that the dendritic fields of the cells do not overlap. These cells sum activities in the colour or texture maps, hence boundary conspicuity at individual pixel positions is reinforced at this level, but also at the next level which deals with local geometry. At the second level, there are many
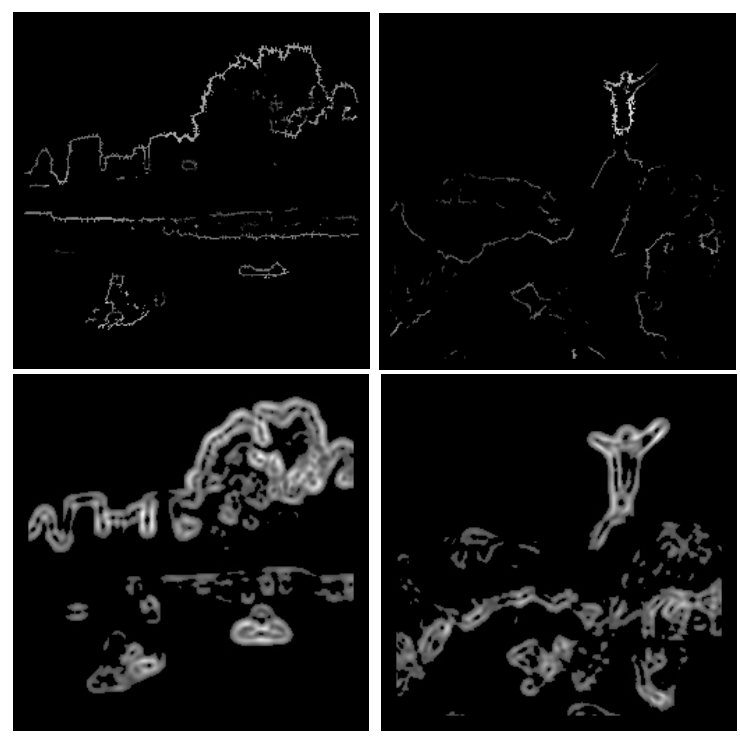

Figure 4: Colour boundary conspicuity (top) for images park (left) and mountain (right). Bottom: texture boundaries.

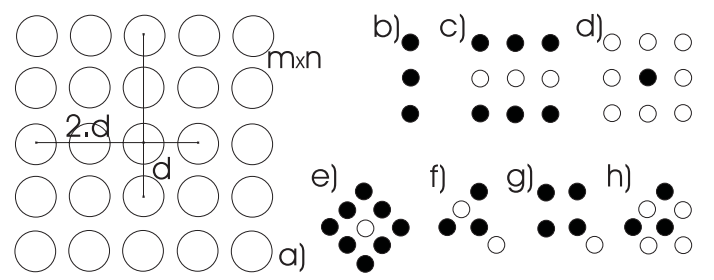

Figure 5: Grouping cells for low-level geometry: (a) cluster of cells with their dendritic fields (circles) on a $5 \times 5$ grid; (b) to (h) show examples of spatial configurations.

grouping cells, each one devoted to one geometric configuration on a $5 \times 5$ grid, but not all axons of the cells at the lower level are used. This allows a simple construction of spatial configurations, as shown in Fig. $5 b$ to h, with up to four rotations, i.e., horizontal, vertical and two diagonal orientations. The solid and open circles in Fig. $5 b$ to h refer to the use of the responses of the underlying summation cells: in case of a solid circle the sum $S$ needs to be positive $(S>0)$, in case of an open circle $S=0$, and responses of all other summation cells on the grid are not used (they are "don't care"). Cells at this level take the maximum of the responses of the excitated grouping cells, but only if the spatial configuration of the non-excitated grouping cells is correct. If the response $R$ of configuration $c$ is $R^{c}$, and if we call the configuration of cells which must be excitated $\Omega_{e}^{c}$ and that of the cells which must not be excitated $\Omega_{n e}^{c}$, with $\Omega_{e}^{c}, \Omega_{n e}^{c} \in \Omega$, the $5 \times 5$ grid, and $\Omega_{e}^{c} \wedge \Omega_{n e}^{c}=0$, then

$$
R^{c}=\max _{i \in \Omega_{e}^{c}} S_{i} \Leftarrow \sum_{j \in \Omega_{n e}^{c}} S_{j}=0 .
$$



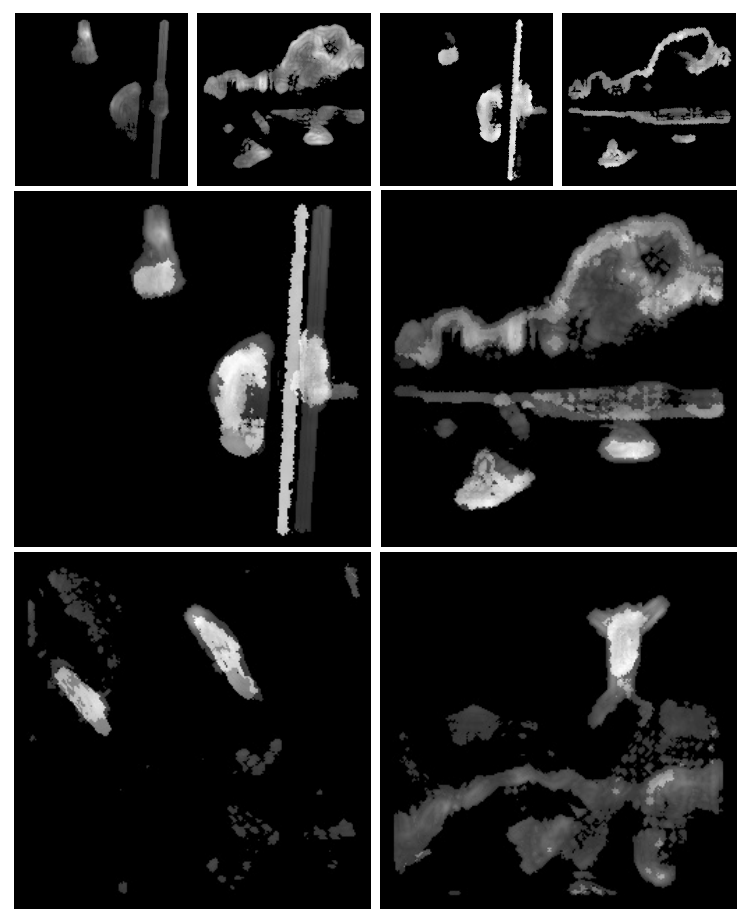

Figure 6: Saliency maps obtained by using only texture boundaries (top-left), only colour conspicuity (top-right), and by combining colour and texture (big images).

The configurations shown in Fig. 5b to h concern, respectively, a line (or an isolated contour), a bar (two parallel contours of a bar), two types of blobs and three types of corners. The $\mathrm{d}$ and e configurations are not rotated, but the other five are (horizontal, vertical and two diagonal orientations), so in total there are 22 configurations $c$ in the total set $C$. Since there may be more configurations valid at the same position, the last cell layer determines the response of the maximum configuration, which yields the saliency map:

$$
R(x, y)=\max _{c \in C} R^{c}(x, y) .
$$

The top row of Fig. 6 shows results obtained when using only texture boundaries (at left), and those when using only colour conspicuity (at right). As can be seen, the maps are different but they complement each other, i.e., texture in general yields more diffuse areas (park and mountain images) whereas colour conspicuity is more concentrated on contours. Combined results, using texture and colour, of all four images are shown in the lower part of Fig. 6. These final maps were created by taking the sum of the two values of the texture and colour saliency maps at each pixel position. It should be stressed that all images were normalised for visualisation purpose, darker areas corresponding to less saliency and brighter ones to more saliency.
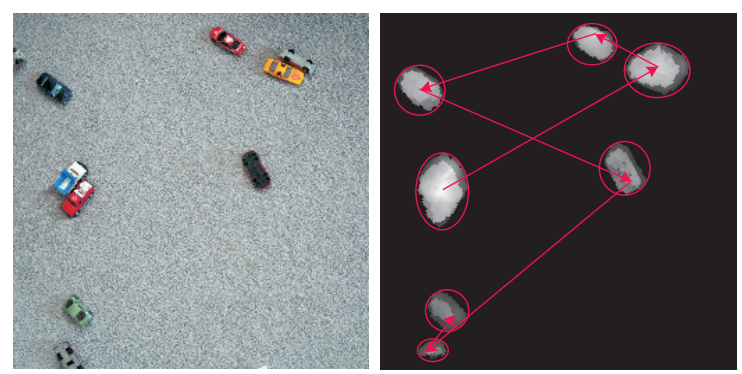

Figure 7: Toy cars image (left) and FoA-driven sequential screening of regions (right).

\section{DISCUSSION}

We introduced a simple, new, and biologically plausible model for obtaining saliency maps based on colour conspicuity and texture boundaries. The model yields very good results in the case of natural scenes. In contrast to the methods employed by Itti and Koch (2001), whose saliency maps are very diffuse versions of entire input images, our method is able to highlight regions, a sort of pre-segregation of complex and conspicuous regions which is later required for precise object segregation in combination with object categorisation and recognition.

The saliency maps provide crucial information for sequential screening of image regions for object recognition and tracking: FoA by fixating conspicuous regions, from the most important regions to the least important ones. Figure 7 shows an input image with toy cars (left), the saliency map (right), and the order, indicated by arrows, in which the regions will be processed. Fixation points were selected automatically by determining the highest response in the saliency map within each region, and regions are fixated using inhibition-of-return. Despite the fact that saliency based on texture boundaries is more diffuse than that on the basis of colour conspicuity, car-region segregation is rather precise. The main reason for this precision is that low-level geometry processing mainly occurs at contours and inside objects, i.e., it does not lead to region growing.

The saliency model is now being extended by motion and disparity information, after which it can be integrated into a complete architecture for invariant object categorization and recognition (Rodrigues and du Buf, 2006, 2007), which is based on multi-scale keypoints, lines and edges derived from responses of cortical simple, complex and end-stopped cells. This is beyond the scope of this paper, but, as mentioned in the Introduction, very fast global and local gist vision are two basic building blocks of an integrated system. Until here, low-level geometry processing has only been used for producing saliency maps for FoA with segregated regions. But since low-level ge- 


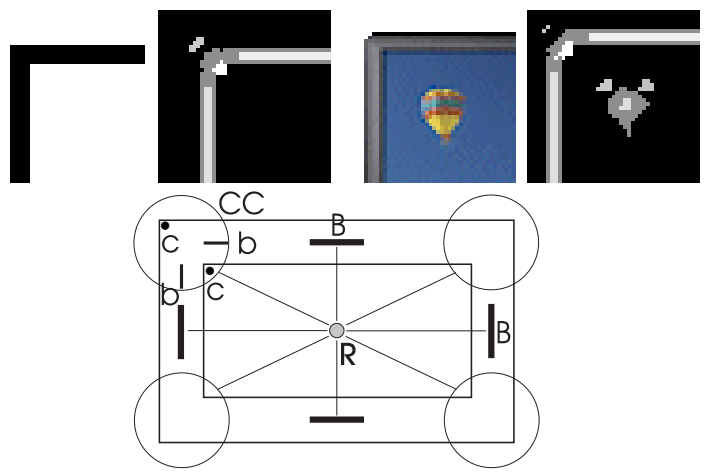

Figure 8: Low-level geometry (top) and example of midand high-level geometry groupings (bottom); see text for details.

ometry information has already been extracted, it is therefore available for obtaining local object gist, for example providing cues which are used for a first and fast selection of possible object categories in memory (Bar M., et al., 2006). This is a purely bottom-up and data-parallel process for bootstrapping the serial object categorization and recognition processes which are controlled by top-down attention.

Low-level geometry is difficult to visualise, because it consists of a large number of spatial maps, in this paper limited to 22 but there could be more, one for each spatial configuration. Figure 8 (top), shows detail images with a few configurations coded by different levels of gray, i.e., corners, bars and blobs. The input was an ideal rectangle with two sharp corners (left) and a computer monitor with a sharp inner corner but rounded outer one. Despite the outer corner being rounded, evidence for a corner has been detected at two pixel positions. These results were obtained on the basis of colour conspicuity, but later texture and colour information should be combined, and low-level geometry should be used to construct midand high-level geometry. The latter idea is illustrated in Fig. 8 (bottom): at low level corners (c) and bars (b) are detected. At mid level, these can be grouped into a complex corner (CC), and at high level the CCs, together with linking bars B, into a rectangle R. Such an $\mathrm{R}$ structure is typical for man-made objects, for example a computer monitor or a photo frame. This example of high-level geometry is perhaps the last level below semantic processing: a computer monitor in combination with one or more photo frames is an indication for global scene gist: our office. In any case, the large number of features at the lowest level (64 Gabor channels) is reduced to the number of spatial configurations at low-level geometry, here 22. Groupings at mid level (e.g., complex corner CC) may lead to less configurations, but at high level (e.g., rectangle $\mathrm{R}$ ) the number of configurations will increase again, be- cause many elementary shapes must be represented. On the other hand, the precise localisation of configurations which is required at low level is not necessary at higher levels; for example, grouping cells for complex corners CC may be located somewhere near the centres of the circles in Fig. 8 (bottom), as long as their dendritic fields are big enough to receive input from two corner and two bar cells. These aspects are subject to further research.

\section{ACKNOWLEDGEMENTS}

Research supported by the Portuguese Foundation for Science and Technology (FCT), through the pluriannual funding of the Inst. for Systems and Robotics through the POS_Conhecimento Program (includes FEDER funds), and by the FCT project SmartVision: active vision for the blind (PTDC/EIA/73633/2006).

\section{REFERENCES}

Bar M., et al. (2006). Top-down facilitation of visual recognition. PNAS, 103(2):449-454.

du Buf, J. (2007). Improved grating and bar cell models in cortical area V1 and texture coding. Image and Vision Computing, 25(6):873-882.

Elazary, L. and Itti, L. (2008). Interesting objects are visually salient. Journal of Vision, 8(3):1-15.

Finlayson, G., Schiele, B., and Crowley, J. (1998). Comprehensive colour image normalization. Proc. 5th Europ. Conf. Comp. Vision, I:475-490.

Hubel, D. (1995). Eye, brain and vision. Scientific American Library.

Itti, L. and Koch, C. (2001). Computational modeling of visual attention. Nature Reviews: Neuroscience, 2(3):194-203.

Niebur, E. and Koch, C. (1996). Control of selective visual attention: Modeling the 'where' pathway. Neural Information Processing Systems, 8:802-808.

Rensink, R. (2000). The dynamic representation of scenes. Visual Cogn., 7(1-3):17-42.

Rodrigues, J. and du Buf, J. (2004). Visual cortex frontend: integrating lines, edges, keypoints and disparity. Proc. Int. Conf. Image Anal. Recogn., Springer LNCS 3211(1):664-671.

Rodrigues, J. and du Buf, J. (2006). Multi-scale keypoints in V1 and beyond: object segregation, scale selection, saliency maps and face detection. BioSystems, 2:7590.

Rodrigues, J. and du Buf, J. (2007). Invariant multiscale object categorisation and recognition. Proc. 3rd Iberian Conf. on Patt. Recogn. and Image Anal., Springer LNCS 4477:459-466.

van de Weijer, J., Gevers, T., and Bagdanov, A. D. (2006). Boosting color saliency in image feature detection. IEEE Tr. PAMI, 28(1):150-156. 